\title{
Effects of magnetic fields on quark-antiquark interactions
}

\author{
Claudio Bonati, Massimo D’Elia, Marco Mariti, Michele Mesiti, Francesco Negro, \\ Andrea Rucci ${ }^{\dagger}$ \\ Department of Physics of University of Pisa and INFN Pisa \\ E-mail: bonati@df.unipi.it, delia@df.unipi.it, mariti@df.unipi.it, \\ mesiti@pi.infn.it, fnegro@pi.infn.it, andrea.rucci@pi.infn.it
}

\section{Francesco Sanfilippo}

School of Physics and Astronomy of University of Southampton, United Kindgdom

E-mail: fr.sanfilippo@gmail.com

\begin{abstract}
We discuss some recent results obtained in the study of strong quark-antiquark interactions in the presence of intense external magnetic fields by means of lattice QCD simulations. We confirm previous findings and show that both at zero and finite temperature the external field induces anisotropies in the static quark potential. An in-depth study suggests that the effects are essentially due to the variation of the string tension whose angular dependence can be nicely parametrized by the first allowed term in a Fourier expansion. In the confined phase at high temperature, we observe that the suppression of the string tension is enhanced as the strength of the external field increases. Our results support the idea that the loss of confining properties is the dominant effect related to the decrease of $T_{c}$ as a function of $B$.
\end{abstract}

34th annual International Symposium on Lattice Field Theory

24-30 July 2016

University of Southampton, $U K$

\footnotetext{
*Present address: Dipartimento di Fisica e Astronomia \& INFN Sez. Firenze, Via Sansone 1, 50019, Sesto Fiorentino (FI), Italy

${ }^{\dagger}$ Speaker.
} 


\section{Introduction}

In many situations, such as heavy ion collisions [1] or in the early Universe [2], large magnetic fields with intensities of $|e| B \gtrsim m_{\pi}^{2}$ are produced and affect the properties of strongly interacting matter. In these contexts, the magnetic fields may lead to significant modifications of the properties of QCD. Indeed, even if gluons are not directly coupled to electro-magnetic fields, substantial contributions can arise from quark loops. This is confirmed both by analytical studies that use effective field theories or perturbation theory and by many evidences from lattice investigations (see, e.g., Refs. [3, 4] for general reviews).

Important modifications may be expected on the static potential between heavy quarks. In the explorative study in Ref. [5] it has been found that both the Coulomb term and the linear confining piece of the potential become anisotropic when a strong external magnetic field is turned on. This has also been predicted by several model studies and could have phenomenologically relevant effects in the heavy meson spectrum and production in heavy ion collision [6-10]. In our study we carry on the investigation of these effects on the potential at zero temperature and explore also the confined phase in the finite temperature regime. At $T=0$, a quantitative description of the behaviour of the string tension and of its angular dependence is provided, together with hints about its fate in the large field limit. Then we move to the exploration of the $T>0$ regime, in order to check whether the anisotropic effect survives, and also to investigate the role of the magnetic field in the string tension suppression near the deconfinement transition.

\section{Numerical setup}

In our work we consider QCD with $N_{f}=2+1$ quark flavours discretized using a Symanzik tree-level improved gauge action and stout improved rooted staggered fermions. The partition function reads

$$
Z(B)=\int \mathscr{D} U e^{-\mathscr{S}_{Y M}} \prod_{f=u, d, s} \operatorname{det}\left(D_{\mathrm{st}}^{f}[B]\right)^{1 / 4} .
$$

where $\mathscr{D} U$ is the measure over the gauge link variables, $\mathscr{S}_{Y M}$ is the gauge action $[11,12]$ involving the real part of the traces over $1 \times 1$ and $1 \times 2$ loops and $D_{\text {st }}^{f}[B]$ is the staggered fermion matrix with two-times stout-smeared gauge links [13].

In order to consider the effect of an external background magnetic field, in the continuum we introduce the abelian gauge four-potential $a_{\mu}$ into the quark covariant derivative which takes the form $D_{\mu}=\partial_{\mu}+i g A_{\mu}^{a} T^{a}+i q_{f} a_{\mu}$ with $q_{f}$ electric charge related to the quark flavour $f$. In the discretized theory, where this operator is written in terms of the $\mathrm{SU}(3)$ gauge links $U_{i: \mu}$ (with $i$ lattice site and $\mu$ direction), the insertion of the electromagnetic field corresponds to the substitution $U_{i: \mu} \rightarrow u_{i: \mu} U_{i: \mu}$ where $u_{i: \mu}$ is a $\mathrm{U}(1)$ phase related to $a_{\mu}$. We consider external fields, hence $a_{\mu}$ is non-propagating: no $a_{\mu}$ kinetic term is added to the Lagrangian.

In our case we work with a constant and uniform magnetic field [3]. If we choose to fix $\vec{B}$ along one of the lattice axes (say $\hat{z}$ ) then it is possible to show that, due to the lattice periodic boundary conditions, the quantization condition $e B_{z}=6 \pi b_{z} /\left(L_{x} L_{y}\right)$ with $b_{z} \in \mathbb{Z}$ must hold, where $e$ is the electric charge unity and $L_{x}$ and $L_{y}$ are the lattice extents along $\hat{x}$ and $\hat{y}$. If $\vec{B}$ is not aligned along one of the axes, we can consider each component separately and write the total abelian phase 
as the product of them over the three axes [14]. Each component of $\vec{B}$ must satisfy an independent quantization condition and $\vec{B}$ will be associated to an integer vector $\vec{b}$. If $L_{x}=L_{y}=L_{z}$ the magnetic field vector $\vec{B}$ is proportional to $\vec{b}$.

We performed simulations at physical quark masses, i.e. tuning the parameters according to the line of constant physics reported in Refs. $[15,16]$. Note that the presence of the external field does not affect the lattice spacing [17]. At zero temperature we used four lattices $24^{4}, 32^{4}, 40^{4}$ and $48^{3} \times 96$ with spacings ranging from $a=0.2173 \mathrm{fm}$ to $a=0.0989 \mathrm{fm}$, and for each of them, we performed runs with magnetic field quanta up to $|\vec{b}|=40$; this give us access to fields roughly up to $|e| B \sim 1 \mathrm{GeV}^{2}$. For $T>0$ we used lattices $48^{3} \times N_{t}$ with $N_{t}=\{20,16,14\}$ and $a=0.0989 \mathrm{fm}$, corresponding to physical temperatures sligthly below $T_{c}$. In all cases, gauge configurations have been sampled by the Rational Hybrid Monte-Carlo algorithm [18], collecting statistics of the order of $10^{3}-10^{4}$ trajectories for each value of $\vec{b}$.

At $T=0$, the static $Q \bar{Q}$ potential $V(r)$ is extracted from planar Wilson loops. Note that loops oriented along different directions cannot generically be averaged since the $O(3)$ rotational symmetry is broken to $O(2)$ by the external magnetic field. At finite $T$, instead, we extracted the free energy $F_{Q \bar{Q}}(r, T)$ of a quark-antiquark pair from Polyakov loop correlators.

\section{Analysis and results}

We adopted the following strategy: first, we determined the values of the potential parameter at $T=0$ and $|e| B=0$ in order to use them as a reference for the subsequent analysis. Then we investigated the influence of the external field, performing a continuum limit extrapolation of all the relevant observables. Finally, we moved on to the $T>0$ regime, by working on our finest lattice to investigate whether the $T=0$ anisotropies survive.

\section{1 $\mathrm{T}=\mathbf{0}$}

It is known that the $Q \bar{Q}$ potential can be well described by the Cornell parametrization [19]

$$
V(r)=-\frac{\alpha}{r}+\sigma r+V_{0},
$$

where $\alpha$ is the Coulomb term, $\sigma$ is the so-called string tension and $V_{0}$ is a constant. This functional form fits very well our data at $T=0$ and $B=0$ with parameters ${ }^{1} \alpha=0.395(22)(26)$ and $\sqrt{\sigma}=$ $448(20)(09) \mathrm{MeV}$, that will be used as reference in the following, corresponding to a value of the Sommer parameter [20] $r_{0}=0.489(20)(04) \mathrm{fm}$.

Turning the magnetic field on, we expect the potential to become anisotropic and to acquire different values in the directions orthogonal or parallel to $\vec{B}$ [5]. In general, one can promote the potential $V(r)$ to a function $V(r, \theta, B)$ of the magnetic field and of the angle $\theta$ between $\vec{B}$ and the $Q \bar{Q}$ orientation. This general parametrization takes into account the residual cylindrical symmetry around $\vec{B}$; we will also impose symmetry under the reflection $\vec{B} \rightarrow-\vec{B}$. Then, assuming the potential to be of the Cornell form (3.1) for all values of $\theta$, the most general form of $V$ compatible with

\footnotetext{
${ }^{1}$ The statistical error is obtained from the $\mathscr{O}\left(a^{2}\right)$ fit of our data (the largest lattice spacing has been discarded); the systematic uncertainty has been estimated by using also the $\mathscr{O}\left(a^{4}\right)$ fit model.
} 

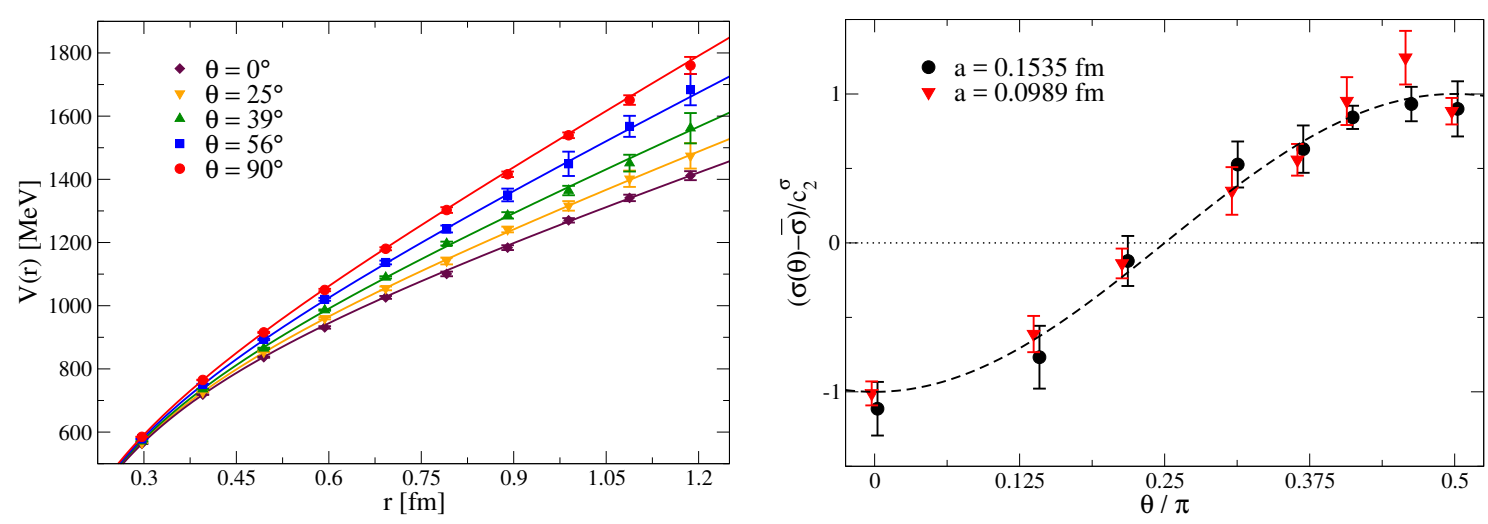

Figure 1: Left: the $Q \bar{Q}$ potential from the $48^{3} \times 96$ lattice for some values of $\theta$ at $|e| B \simeq 1 \mathrm{GeV}^{2}$. Right: angular variation of the string tension compared with the function $-\cos (2 \theta)$ (dashed line).

these symmetries is

$$
V(r, \theta ; B)=-\frac{\alpha(\theta ; B)}{r}+\sigma(\theta ; B) r+V_{0}(\theta ; B) \quad \mathscr{O}(\theta ; B)=\left(1-\sum_{n \geq 1} c_{2 n}^{\mathscr{O}}(B) \cos (2 n \theta)\right) \overline{\mathscr{O}}
$$

where the potential parameters $\mathscr{O}=\left\{\alpha, \sigma, V_{0}\right\}$ bring all the dependence on $\vec{B}$.

In order to verify the reliability of this model, we analyzed the angular dependence of the static potential on our two finest lattices (corresponding to the lattice sizes $48^{3} \times 96$ and $32^{4}$ ) for three different orientations of the magnetic field with $|b| \simeq 32$, giving us access to 8 different $\theta$ angles. Results are shown in Fig. 1. As expected from the preliminary data in Ref. [5], the potential $V(r)$ increases with the angle, and the model in Eq. (3.2) fits very well our data. We found that the values $\overline{\mathcal{O}}$, which are a sort of average of the parameters over the angles, are all compatible with those at $B=0$. Morover, it turns out that it is sufficient to consider only the first terms $c_{2}^{\mathscr{O}}$ in the Fourier series $^{2}$, as show in Fig. 1.

We performed a continuum limit extrapolation to determine whether these anisotropies are only lattice artifacts or truly physical effects. A complete determination of the angular distributions and of the $\vec{B}$ dependence would require many simulations with different lattice spacings and several values and orientations of the magnetic field. However, since only the $c_{2} \mathrm{~s}$ terms are needed in the description of the angular dependence (at least with our precision), we can simplify the task considerably by considering the two quantities

$$
\delta^{\mathscr{O}}(|e| B)=\frac{\mathscr{O}_{X Y}(|e| B)-\mathscr{O}_{Z}(|e| B)}{\mathscr{O}_{X Y}(|e| B)+\mathscr{O}_{Z}(|e| B)} \quad R^{\mathscr{O}}(|e| B)=\frac{\mathscr{O}_{X Y}(|e| B)+\mathscr{O}_{Z}(|e| B)}{2 \mathscr{O}(|e| B=0)} .
$$

These are, respectively, the anisotropy and the average value of the parameter $\mathscr{O}$ taken along the $\hat{x}, \hat{y}$ and $\hat{z}$ directions. Using the fact that $c_{2 n} \simeq 0$ when $n>1$, it is easy to show that $\delta^{\mathscr{O}}(|e| B) \simeq c_{2}^{\mathscr{O}}(|e| B)$ and $R^{\mathscr{O}}(|e| B) \simeq \overline{\mathscr{O}}(|e| B) / \mathscr{O}(|e| B=0)$. Therefore, we can access all the information about the anisotropies just by measuring the potential in the directions parallel or orthogonal to the magnetic field.

Results for the anisotropies of $\alpha$ and $\sigma$, together with their continuum limit extrapolations, are shown in Fig. 2. The fit procedure has been performed using a general power law for the

\footnotetext{
${ }^{2}$ If successive terms $c_{2 n}$ with $n>1$ are included in the fit model, they turn out to be all compatible with zero.
} 

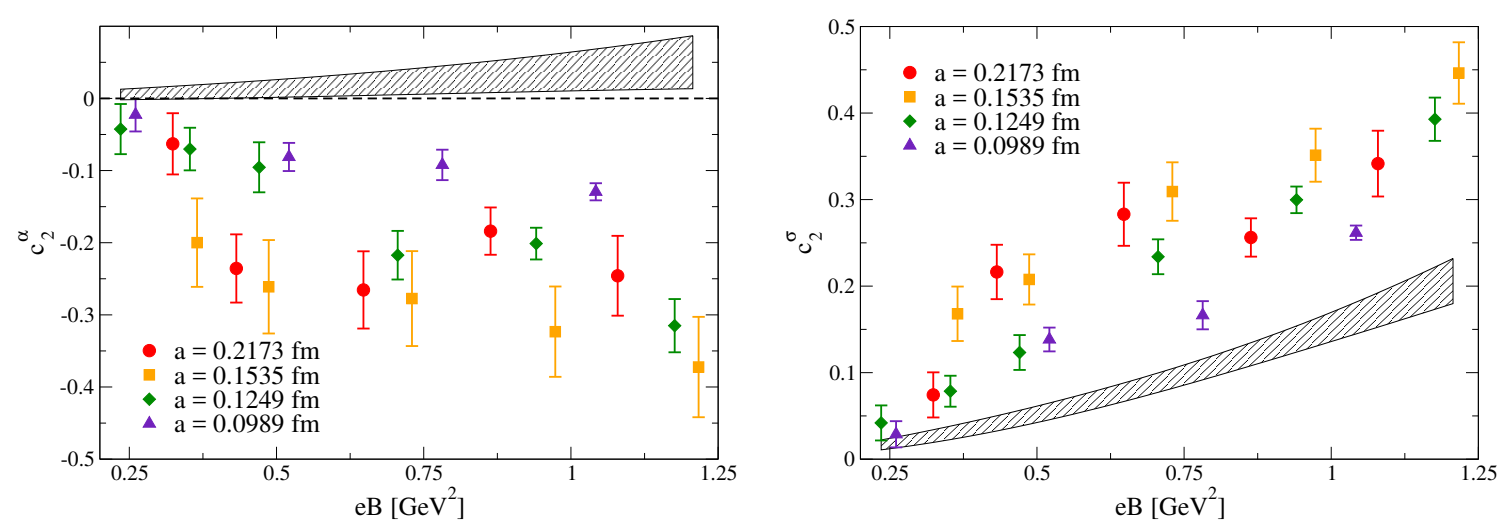

Figure 2: The coefficients $c_{2}^{\mathscr{O}}$ of $\alpha$ (left) and $\sigma$ (right). Bands are obtained through a continuum limit extrapolation on the data of our three finest lattices.

coefficients as a function of $|e| B$, with $\mathscr{O}\left(a^{2}\right)$ corrections, and it gave us very good values of the $\chi^{2}$ test [14]. In the case of the string tension, the anisotropy $c_{2}^{\sigma}$ survives in the continuum limit and grows with the magnetic field. Conversely, the continuum limit of $c_{2}^{\alpha}$ is compatible with zero, like for $c_{2}^{V_{0}}$ [14]. We can therefore conclude that the effect of the magnetic field on the static potential persists in the continuum limit and is mostly due to the variation of the string tension.

Finally, our findings suggest a sort of anisotropic deconfinement at very large magnetic fields (see Fig. 3). Indeed, for $|e| B \sim 4 \mathrm{GeV}^{2}$ the string tension is predicted to vanish in the longitudinal direction by extrapolating our fit results. Data obtained for large $|e| B$ seem to roughly agree with this behaviour, however a precise continuum extrapolation is difficult in this region due to large cut-off effects expected at $|e| B \sim a^{-2}$. By now, the presence of this sort of critical value of $|e| B$ is to be taken as a fascinating speculation.

\section{2 $\mathrm{T}>0$}

Results for the free energy of a static $Q \bar{Q}$ pair at temperatures lower than $T_{c} \sim 155 \mathrm{MeV}$ are shown in Fig. 4. As one can see, the potential roughly follows the same behaviour observed in

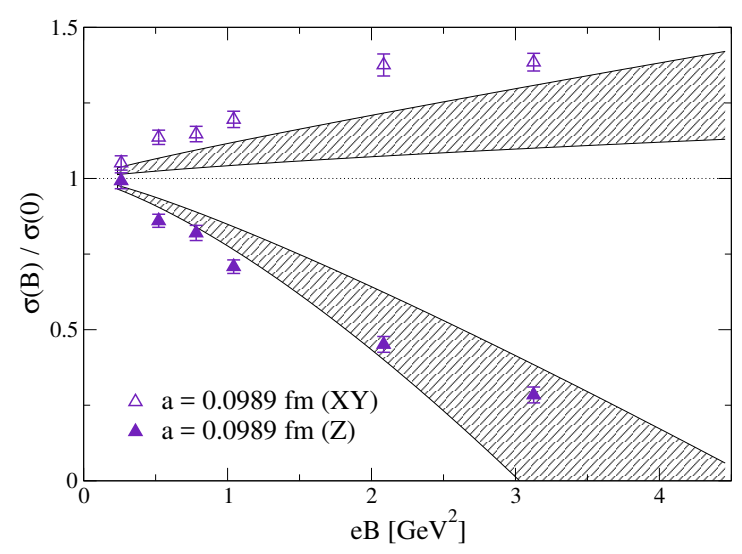

Figure 3: String tension as a function $|e| B$. Bands are obtained through a continuum limit extrapolation performed only in the range $|e| B \lesssim 1 \mathrm{GeV}^{2}$. 

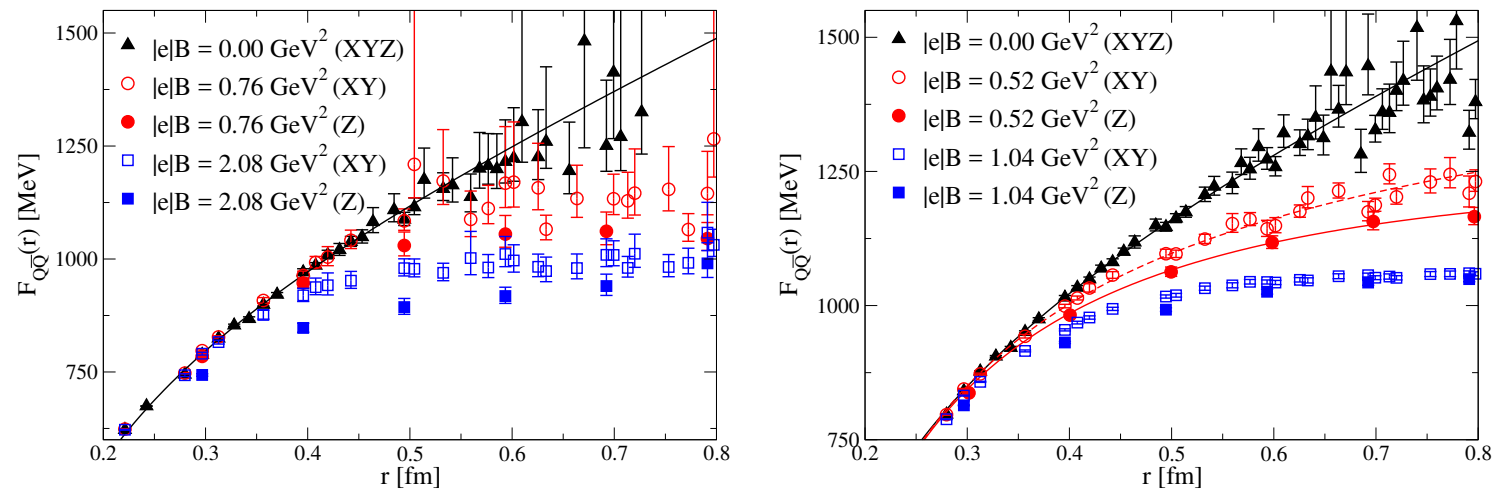

Figure 4: Free energy $F_{Q \bar{Q}}(r, T)$ from $48^{3}$ lattices for two different temperature $T \simeq 100 \mathrm{MeV}$ (left) and $T \simeq 125 \mathrm{MeV}$ (right). Curves are obtained fitting the Cornell form and are shown only in the cases in which this parametrization reveals to be a good description of the data.

the $T=0$ case, with the anisotropy still present. In this temperature regime, the dominant effect turns out to be the flattening of the potential as $B$ is increased. As a result, the Cornell form in Eq. (3.1) ceases being suitable for the description of the potential when both the temperature and the magnetic field grow. This can be also seen from the behaviour of string tension (leftmost plot in Fig. 5): as expected, $\sigma$ tends to vanish as $T$ reaches the pseudocritical temperature $T_{c}$, but this effect is remarkably enhanced by the presence of the magnetic field.

These results are consistent with the picture of a decreasing chiral pseudocritical temperature shown in previous studies [17, 21]. In the so-called inverse magnetic catalysis, the decrease of $T_{c}$ is responsable of the non-monotonic behaviour of the chiral condensate near the transition temperature. Our data gives us evidences of the same phenomenon at the level of the confining properties. Morover, the latter seems to be dominant effect: in the range of $B$ and $T$ explored (see right plot Fig. 5), the inverse magnetic catalysis is hardly noticeable, while the flattening of the potential is clearly visible.
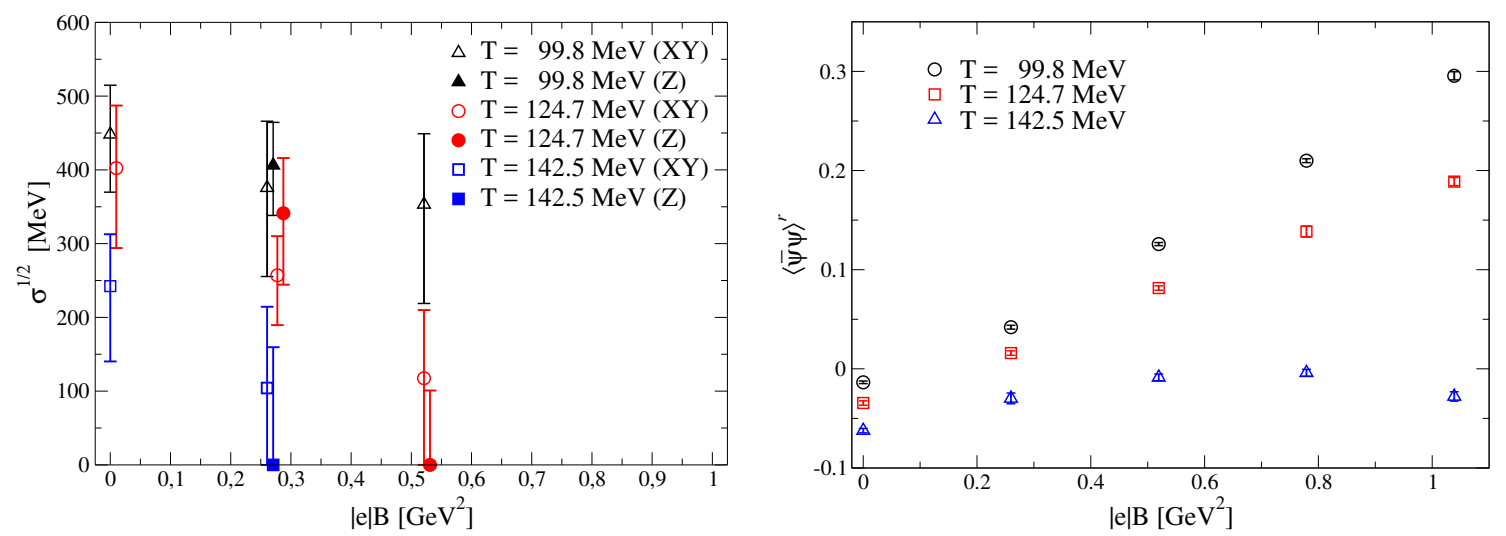

Figure 5: Square root $\sqrt{\sigma}$ of the string tension (left) and the renormalized chiral condensate [17] (right) as function of the magnetic field for some temperatures below $T_{c}$. 


\section{Conclusions}

It has been confirmed that the static $Q \bar{Q}$ potential is strongly affected by an external magnetic field, becoming anisotropic. Studying its angular dependence and performing continuum extrapolations at $T=0$ we have shown that the anisotropy is a physical effect that persists in the continuum limit. This is mostly due to the variation of the string tension, whose angular dependence is very well described by just the first Fourier term $c_{2}$. In the high-temperature regime, the anisotropy is reduced and the main effect of the magnetic field is the precocious disappeareance of the confining properties, indicated by the flattening of the potential at large distances and by the vanishing of the string tension, something that can be called deconfinement catalysis. This phenomenon can be understood in terms of a decreasing $T_{c}$ and is noticeable even at low values of the magnetic field, when there are no signals of inverse magnetic catalysis in the chiral condensate.

\section{Acknowledgments}

We acknowledge PRACE for giving us access to FERMI machine at CINECA in Italy, under project Pra09-2400 - SISMAF. FS received funding from the European Research Council of the European Community Seventh Framework Programme (FP7/2007-2013) ERC grant agreement No 279757. FN acknowledges financial support from the INFN project SUMA.

\section{References}

[1] V. Skokov, A. Y. Illarionov and V. Toneev, Int. J. Mod. Phys. A 24, 5925 (2009) [nucl-th/0907.1396].

[2] T. Vachaspati, Phys. Lett. B 265 (1991) 258.

[3] D. E. Kharzeev, K. Landsteiner, A. Schmitt and H. U. Yee, Lect. Notes Phys. 871, 1 (2013).

[4] V. A. Miransky and I. A. Shovkovy, Phys. Rept. 576, 1 (2015) [hep-ph/1503.00732].

[5] C. Bonati et al., Phys. Rev. D 89, 114502 (2014) [hep-lat/1403.6094].

[6] K. Hattori, T. Kojo and N. Su, Nucl. Phys. A 951, 1 (2016) [hep-ph/1512.07361].

[7] X. Guo, S. Shi, N. Xu, Z. Xu and P. Zhuang, Phys. Lett. B 751, 215 (2015) [hep-ph/1502.04407].

[8] K. Fukushima, K. Hattori, H. U. Yee and Y. Yin, Phys. Rev. D 93, 074028 (2016) [hep-ph/1512.03689].

[9] P. Gubler et al. Phys. Rev. D 93, 054026 (2016) [hep-ph/1512.08864].

[10] C. Bonati, M. D’Elia and A. Rucci, Phys. Rev. D 92, 054014 (2015) [hep-ph/1506.07890].

[11] P. Weisz, Nucl. Phys. B 212, 1 (1983).

[12] G. Curci, P. Menotti and G. Paffuti, Phys. Lett. B 130, 205 (1983)

[13] C. Morningstar and M. J. Peardon, Phys. Rev. D 69, 054501 (2004) [hep-lat/0311018].

[14] C. Bonati et al., Phys. Rev. D 94 (2016) 094007 [hep-lat/1607.08160].

[15] Y. Aoki et al., JHEP 0906, 088 (2009) [hep-lat/0903.4155].

[16] S. Borsanyi et al., JHEP 1011, 077 (2010) [hep-lat/1007.2580].

[17] G. S. Bali et al., JHEP 1202, 044 (2012) [hep-lat/1111.4956 [hep-lat]].

[18] M. A. Clark and A. D. Kennedy, Phys. Rev. D 75, 011502 (2007) [hep-lat/0610047].

[19] E. Eichten et al., Phys. Rev. Lett. 34, 369 (1975).

[20] R. Sommer, Nucl. Phys. B 411, 839 (1994) [hep-lat/9310022].

[21] G. S. Bali et al., Phys. Rev. D 86, 071502 (2012) [hep-lat/1206.4205]. 